\title{
P-1037- National Health Education on Diabetes - the practice in China
}

\author{
Wei Wei, Li Fangbo, Chinese Center for Health Education
}
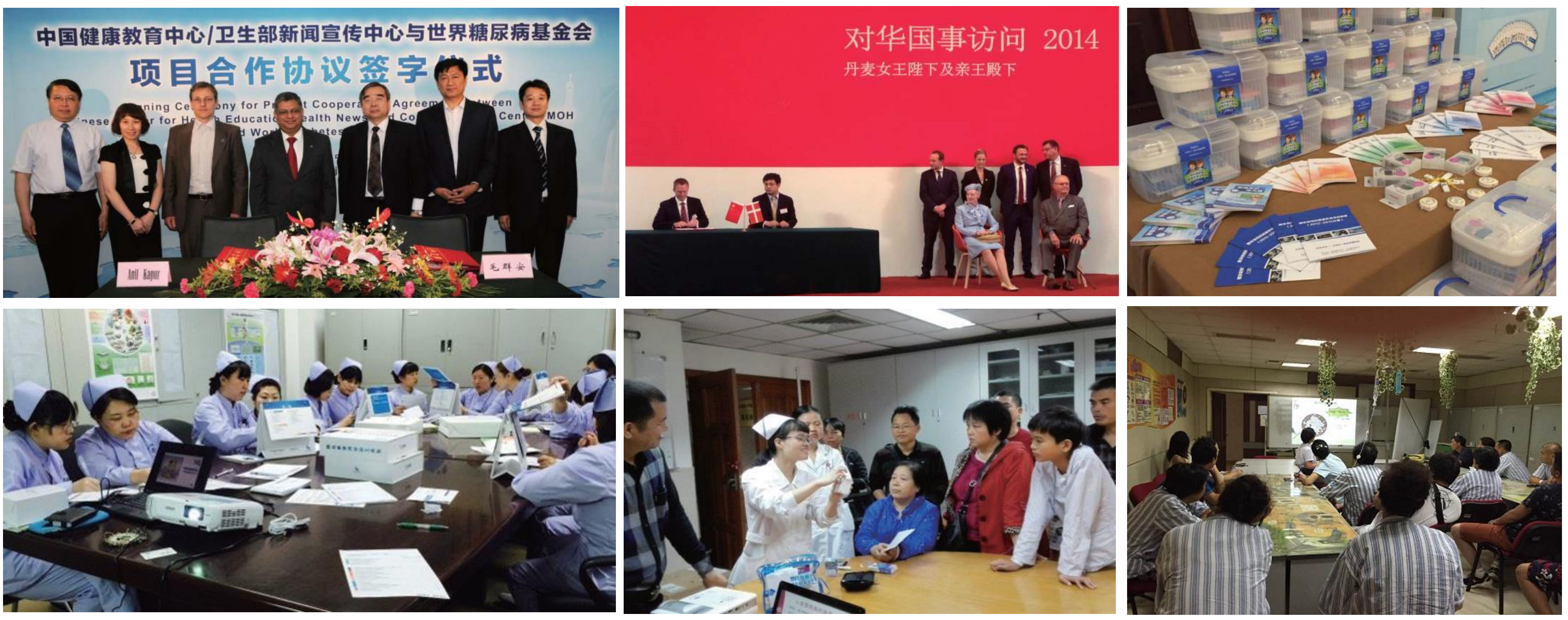

\section{Background}

More than 93 million Chinese adults have diabetes $-9.7 \%$ of the adult population. Around 43 million of these live in rural areas. $63 \%$ of people having diabetes are undiagnosed. 1.1 million Chinese people die each year because of diabetes, which means more than 3,000 people every day.

Fortunately there is compelling evidence from clinical trials that lifestyle modifications and education can minimize the risk of diabetes. Persons with diabetes must understand their disease and be empowered to avoid obesity, smoking and unhealthy diets, encouraged to do exercise and control blood glucose. Good health education, health promotion and access to professional care are essential for persons with diabetes.

\section{Aims}

Raise awareness of diabetes and self-care capacity among Chinese residents, improve the capacity of diabetes management among primary health workers, promote the attention of all levels of government and relevant departments to diabetes prevention and control, and create a public opinion environment that the whole society cares, supports and participates in diabetes prevention and control.

\section{Method}

The whole project lasted for 3 years. In the first year, a baseline survey was conducted to understand the diabetes related KAP among Chinese resident. A total of 4282 people from six provinces in China were surveyed.

Based on the baseline results, a series of intervention activities were carried out to improve the residents' diabetes related KAP.

- Core Information and Patient Self-care Brochure development, best practices collection, health education program production and poster campaign, in order to raise awareness of diabetes, related NCDs and self-care capacity among residents;

- Technical Guide for Diabetes Management development and on field trainings, in order to improve the capacity of diabetes management among primary professionals;

- Leadership engagement meeting and World Diabetes Day public events, in order to promote the attention of all levels of government and relevant departments about diabetes prevention and control;

[1] IDF Diabetes Atlas 6th Edition (2013)

[2] GUO Xiaohui, YUAN Li, LOU Qingqing, SHEN Li, etal. Chinese Diabetes Education Status Survey Study

Group. A nationwide survey of diabetes education, self-management and glycemic control in patients with

type 2 diabetes inChina [J]. Chinese Medical Journal, 2012;125(23):4175-4180.

[3] Chinese diabetes society. Standards of care for type 2 diabetes in China (2013).
- Media Coverage Guide for Diabetes Prevention development and journalists training, in order to create a public opinion environment that the whole society cares, supports and participates in diabetes prevention and control.

- In the third year, the final assessment among 2700 patients was conducted to evaluate the project effects.
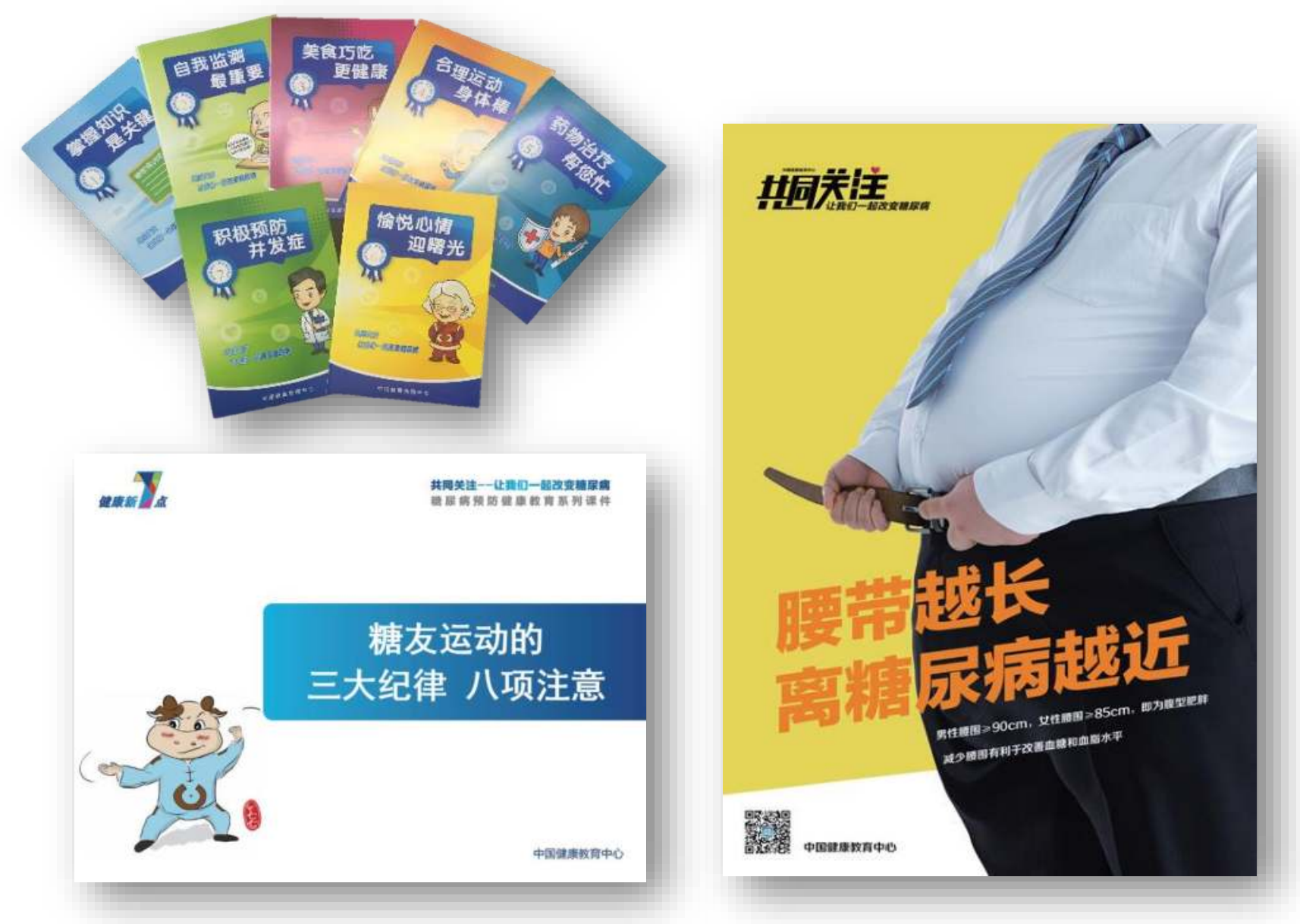

\section{Results}

The project improved the patients' self-management behaviors: the proportion of "Follow doctors' advice to take the drug/insulin" was raised from $62.6 \%$ to $67.5 \%$; the proportion of "Alimentary control" was raised from $71.0 \%$ to $81.3 \%$; the proportion of "Sports exercise" was raised from $76.4 \%$ to $87.1 \%$; the proportion of "Blood glucose monitoring" was raised from $43.7 \%$ to $48.5 \%$.

\section{Discussion}

Although the patients' self-care behavior was improved, but the proportion of some behaviors is still not satisfied. As the next step plan, we suggested that we should help the primary professionals to improve their capacity of diabetic patients' management, and guide them to develop the health management on diabetic patients. 